\title{
Advances in Aflatoxins Monitoring of Stored Food
}

\author{
Mariana Matulovic da Silva Rodrigueiro ${ }^{1}{ }^{\text {D }}$, Cleber Alexandre de Amorim ${ }^{1}$ (D), Kassandra Sussi Mustafé \\ Oliveira $^{2}$ (D), Flávio José de Oliveira Morais ${ }^{1(D)}$, Priyanka Joshi ${ }^{3}$ (D), Kate Cristina Blanco ${ }^{3 * *(D)}$ \\ 1 School of Sciences and Engineering, Av. Domingos da Costa Lopes, São Paulo State University (Unesp), 780 Jardim \\ Itaipu, CEP 17602-496 Tupã, SP, Brazil \\ 2 Faculty of Agricultural Sciences, São Paulo State University (Unesp), Botucatu, São Paulo, Brazil \\ 3 São Carlos Institute of Physics, ,University of São Paulo - Box 369, 13566-970, São Carlos - SP, Brazil \\ * Correspondence: blancokate@gmail.com, kateblancokate@ifsc.usp.br (K.C.B.).
}

Scopus Author ID 35320859700

Received: 30.07.2021; Revised: 9.08.2021; Accepted: 14.08.2021; Published: 17.10.2021

\begin{abstract}
This review provides relevant advances in detecting fungal aflatoxins from agro-industrial products during their development, storage, and processing. For real-time monitoring of these toxins, methodologies were modified and adapted over time. The description of the current literature and the evolution of detection and quantification of aflatoxin in agro-products contaminated by fungi is carried out with the description, mainly of chromatographic, immunochemical, spectroscopic, and genomic methods. In addition to equipment, several methodologies were developed and adapted to improve the specificity of mycotoxin detection through faster analyzes focusing on food safety.
\end{abstract}

Keywords: agricultural products; mycotoxins; aflatoxin; detection; food.

(C) 2021 by the authors. This article is an open-access article distributed under the terms and conditions of the Creative Commons Attribution (CC BY) license (https://creativecommons.org/licenses/by/4.0/).

\section{Introduction}

It is known that microbial toxins have been causing damage to human health through cumulative effects, mainly by fungi. As microbial selection characteristics, when there is an increase in its biomass, metabolites are produced that can confer mutagenic advantages to each species or strain [1]. Fungi from grain and cereal crops have shown adaptive changes to assimilate different types of nutrients and thus become a threat to crops. The fungal aflatoxins of Aspergillus flavus, A. parasiticus, and A. nomius are commonly found in cereal grains, oilseeds, and nuts. Aflatoxins promote tissue damage infections as well as have cumulative effects in both animals and humans that can progress to serious diseases such as hepatomegaly and liver cancer, classified in group three of evidence of carcinogenicity to animals by the International Agency for Research on Cancer [2].

Analysis of aflatoxins from fungi has been carried out for more than 50 years by various chromatography and immunoassay methods, often using these in combination, which can result in increased detection sensitivity and process costs.

The identification of the fungal species or fungal toxin can be performed by high or low sensitivity. This will depend on each country's internal regulations for the consumption of aflatoxins B and G (1 and 2), which have already been documented in countries with permission to consume higher levels of toxins.

Nanosensors and protein and antibody coupling were the main advances in the realtime detection of aflatoxins in the development and storage of agro-industrial products. This review includes a summary of aflatoxin methods and sensors and the implementation of new 
possibilities to detect and monitor products in development and stored to preserve food contaminated by aflatoxins, which can be valuable in the development of new methods.

\section{Fungal Toxins}

\subsection{Formation, significance, and types.}

Fungi, organisms - uni and multicellular - incapable of producing their food, develop in aquatic, aerial, and terrestrial environments. Some fungi species under different biological, chemical, physical, and environmental conditions produce, in addition to biomass, primary and secondary metabolites that can be toxic to humans $[1,2]$. Which can be cited: the genetic fitness; temperature and humidity conditions; oxygen, and carbon dioxide concentrations, and nutrients $[3,4]$

Mycotoxins comprise many low molecular weight chemical structures, grouped according to the degree and type of toxicity, namely: acute, chronic, mutagenic, and teratogenic[5]. The main groups of fungi capable of producing mycotoxins are Aspergillus, Penicillium, Fusarium, and Alternaria. While fungal toxins from Fusarium and Alternarium are often classified as "field fungi", the species Aspergillus and Penicillium are "storage fungi" because they produce mycotoxins correlated to agro-industrial storage conditions[6]. According to Singh et al. [7], among the various mycotoxins (aflatoxin and cyclopiazonic acid (ACP)) linked to Aspergillus species considered relevant. Although this review focuses on fungi and mycotoxins linked to aflatoxin production, a brief taxonomy of the most important species of fungal toxins will be outlined.

Fungi of the Fusarium species produce one of the most contaminating mycotoxins in grains such as corn. Depending on an environmental condition that requires a moisture concentration of 20 to $21 \%$ and water activity between 0.80 and 0.90 , the development of Fusarium adapts favorably to the tropical climate, both in field production and in post-harvest conditions (mainly in storage) [8]. Penicillin from Penicillium chrysogenum is one of the most used medications to treat infections caused by some bacteria. Therefore, not all fungal toxins refer to the contamination of animals and humans, but they are also toxic to certain bacteria[9]. This review focuses on contamination linked mainly to human beings, among the most relevant mycotoxins of this genus. Arternaria toxins produce a series of toxins capable of infecting food crops in the post-harvest period, predominant in cereals, sunflower seeds, olives, and fruits. Due to the substantial evidence of the carcinogenic effects of toxins in living organisms, research on the subject is on the rise in academia [9].

\subsection{Chemical, biological, and environmental aspects.}

In chemical terms, aflatoxins "are polycyclic compounds containing a coumarin nucleus fused to a bifuran and a pentanone. These compounds strongly fluoresce in UV light" ([10], 2008, p. 23). An interesting point to be highlighted is that, although both species $-A$. parasiticus and A. Nomius - trigger the development of aflatoxins of types B and $\mathrm{G}$, the morphology of Nomius is similar to that of A. flavus [10]. The names B and $\mathrm{G}$ come from their fluorescence under UV light (blue or green). According to Pitt [11], 50\% of A. flavus isolates produce aflatoxins.

In general, aflatoxin contamination is predominant in the following foods by A. flavus in peanuts, nuts, corn, cereals, and coffee, A. parasiticus in peanuts, A. nomius centers its development in corn and Brazil nuts. Regarding environmental aspects, the availability of 
water available in the substrate (Aw: water activity) of the fungus development can considerably increase its capacity to produce spores and metabolic activity. According to Lacey, the ideal range of Aw-type water activity for aflatoxin proliferation, both for A. flavus and $A$. parasiticus, ranges from 0.83 to 0.87 [12]. Aflatoxins are normally produced between $12^{\circ} \mathrm{C}$ and $46^{\circ} \mathrm{C}$, with specific optimal ranges for each type and $\mathrm{pH}$ in the range of 3.5 to 8.0. Therefore, they can be produced from hot, dry climates to high moisture content and water concentrations. The exposure and ingestion of foods with high concentrations of aflatoxins can cause the illness and death of living organisms, mainly humans and animals [13].

\subsection{Toxicity and impact on humans and animals.}

Investigative studies of acute aflatoxicosis as a cause of the increase in cases of human liver failure began in the 1960s. The carcinogenicity of aflatoxin was first linked to the susceptibility and development of liver tumors in rats and monkeys. In 1963 the results established from tests of carcinogenic activity in rats with peanut aflatoxin from Aspergillus flavus showed that toxin is an active carcinogen for the subcutaneous tissue of the rat [14]. In 1966, ultrastructural and biochemical abnormalities were demonstrated in the liver of rats and monkeys by aflatoxin B1 (ATB1). In 1971, twenty-four young female monkeys who received 0 to $40.5 \mathrm{mg} / \mathrm{kg}$ ATB1 by weight by oral administration had a median lethal dose of $7.8 \mathrm{mg} / \mathrm{kg}$ for 148 hours, which was detected in the brain, liver, kidney, heart, blood, and bile in some of the animals[15]. In 1980, the carcinogenesis of the precursors of the compounds (versicolorin and sterigmatocystin) of aflatoxin biosynthesis was also determined in rainbow trout embryos[14].

An outbreak of aflatoxicosis in India in 1974 was associated with the consumption of maize contaminated with 2-6 mg/day per month of aflatoxin from Aspergillus flavus[16]. From then on, the problem of exposure of humans to high doses of aflatoxins could awaken to cause irreversible damage to the liver by intoxication (aflatoxicosis). The clinical symptoms described in these cases are vomiting, abdominal pain, liver necrosis, and immunodeficiency. The main indication of this lesion is accompanied by the activity of enzymes in the temporal aspect of the development of the disease. The main related enzymes are $\gamma$-glutamyl transpeptidase, aspartate aminotransferase, alkaline phosphatase, sorbitol dehydrogenase, ornithine carbamyl transferase, and isocitric dehydrogenase [16-18].

In the 70s, studies of AFB1's toxicity mechanisms demonstrated mycotoxin hepatocarcinogenicity. AFB1 (AFB1 8,9-oxide, or AFB1 epoxide) covalently binds to DNA, RNA, or proteins and performs toxic effects on cells through enzymatic and chemical synthesis of monooxygenase associated with liver cytochrome P-450 and acid 3 -chloroperbenzoic[17]. The covalent binding of AFB1 with guanine at the N-7 position induces displacement mutations with intercalation of the AFB1 molecule and base substitution mutations resulting in an incorrect DNA repair. The AFB1 epoxide modifies hepatocellular DNA to a large extent and by the toxin metabolites to a lesser extent [17].

Several experimental studies have shown varied mutations of carcinogenic lesions relating to the toxin's chemical structure and the molecular biology of damaged DNA. In the review, the study of the carcinogenic effect was correlated by computational chemistry of the functional groups of the toxin in the conformational model of DNA and during DNA replication in mutation repair situations[19]. Aflatoxin-N7-guanine is an adduct excretion product of aflatoxin-DNA and can serve as a biomarker associated with a high risk of liver cancer (Figure 1). 
The aflatoxins of type B1, B2, G1, G2, M1, and M2 of A. flavus, Aspergillus nomius, and Aspergillus parasiticus have been affecting products such as corn, rice, pasta, peanuts, peanut butter, pistachio, cassava, tobacco, oilseeds, milk, cheese, yogurt in Algeria, Brazil, India, Italy, Malaysia, Kenya, Nigeria, Sierra Leone, Mexico Monte Carlos, Sub-Saharan Africa, Turkey, Uganda and the United States affecting weight loss, reproduction, teratogenesis, immunosuppression, mutagenesis, kidney cancer, liver cancer and death in animals and hepatomegaly, jaundice, hepatitis, cirrhosis, liver cancer, Reye's syndrome, kwashiorkor and immunosuppression in humans [20-22].

Regarding the degree of toxicity of aflatoxin, Smith points out that B1> G1>B2> G2 in addition, of course, to the consequences related to the time of exposure to the toxin, namely: (i) high doses can contribute to the development of acute illnesses and death, mainly linked to liver dysfunctions; (ii) chronic sublethal doses can cause nutritional and immunological problems; and (iii) all doses have a cumulative effect on cancer risk.

\section{Conventional Methods for Determination of Mycotoxins}

\subsection{Analytical methods.}

In 1974 the Walt King method of analysis using densitometric procedures estimated aflatoxin dosages, and regression analysis indicated the concentration of aflatoxin in a population by several variable components[23]. In 1975 a three-part study was carried out to determine the recovery of aflatoxin $\mathrm{M}$ from pasteurized and stored cow's milk by cellulose column and two-dimensional thin layer chromatography with acetone-chloroform as solvent. A search for aflatoxin in California peanuts found 14 to $74 \%$ of aflatoxins B and G determined by an FDA - Food and Drug Administration approved fluorescent light method. In 1976 New Zealand white rabbits immunized with covalent conjugates prepared with AFB1 Ocarboxymethyl oxime produced antibodies that bind to AFB1, and the specificities of the aflatoxin antisera BI, B2a, G1, G2, Q1, P1 were determined by immunoassay, which was sensitive and did not require purification of the samples by chromatography before analysis. In 1980, a systematic investigation was carried out with the conditions employed for iodination after reverse phase HPLC to increase the fluorescence sensitivity of aflatoxins B1 and G1 reaching a maximum sensitivity of $20 \mathrm{pg}$ of B1 injected into the column. In 1985 highly specific IgM monoclonal antibodies to aflatoxins were covalently linked to Sepharose 4B to isolate aflatoxin from the urine of people and animals exposed to the carcinogen in the environment or under laboratory conditions. In 1994 a method to determine aflatoxin M from parmesan cheese was based on the extraction of aflatoxin $\mathrm{M}$ from cheese by chloroform and high-pressure liquid chromatography. Qualitative analytical methods of aflatoxin were compared with column and thin layer chromatography methods, and most were not statistically different. In 1997 the commercial pasteurized milk samples from the Athens market were analyzed for the combination of Enzyme-Linked Immunosorbent Assay (ELISA) and modified HPLC methods for the fast and reliable determination of aflatoxin M. Aflatoxin contamination in feed and food is monitored in developed countries using ELISA and HPLC, which can be expensive for routine use in many developing countries, highlighting alternative approaches such as aflatoxin B1 blue fluorescence and enhanced by the inclusion of $\beta$ - cyclodextrin in the medium, in addition to the yellow pigment production of biosynthetic intermediates in the aflatoxin production pathway that are $\mathrm{pH}$ indicators [24-26]. 
The selective methods can present disadvantages with the generation of residues in chromatographic methods and high analysis costs in permanent chemical bonds in surfaces functionalized with enzymes and antibodies. Immunoassays are highly specific to epitopes but have cross-reactivity due to the specific identification of chemical substances. Aflatoxins are small non-immunogenic molecules capable of being linked to proteins that generate immune responses identified by methods of producing recombinant antibodies [27]. An optical immunosensor (Figure 1(a-b)) is developed to determine aflatoxins in assay systems that generate specific absorbance when bound to antibodies immobilized in a sensitive optical component. The response angle is increased with the increase in the amount of bound aflatoxin.

The need for highly sensitive multi-analyte methods of the chemical diversity of mycotoxins of heterogeneous biological occurrence is increasingly necessary. Methodologies for determining aflatoxin in agricultural products have already been described by sample preparation with liquid-liquid extraction, solid-phase extraction, enzyme immunoassay, accelerated solvent extraction, solid-phase extraction, solid-phase matrix dispersion followed by instrumentation using high-performance liquid chromatography with fluorescence detection, mass spectrometry, capillary electrophoretic methods, microemulsion electrokinetic chromatography, supercritical fluid chromatography, near-infrared spectroscopy, thin layer chromatography, protein c-reactive, and Fourier transform infrared spectroscopy [28-32].

$\mathrm{R}$

(a)

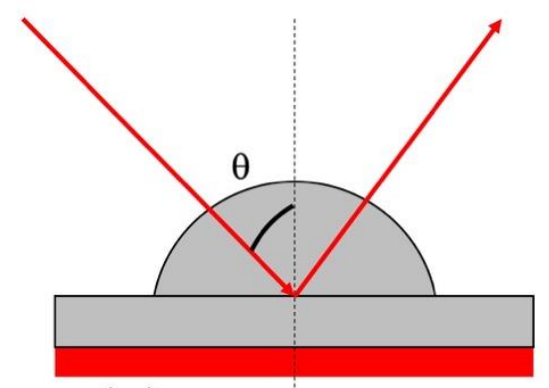

(b)
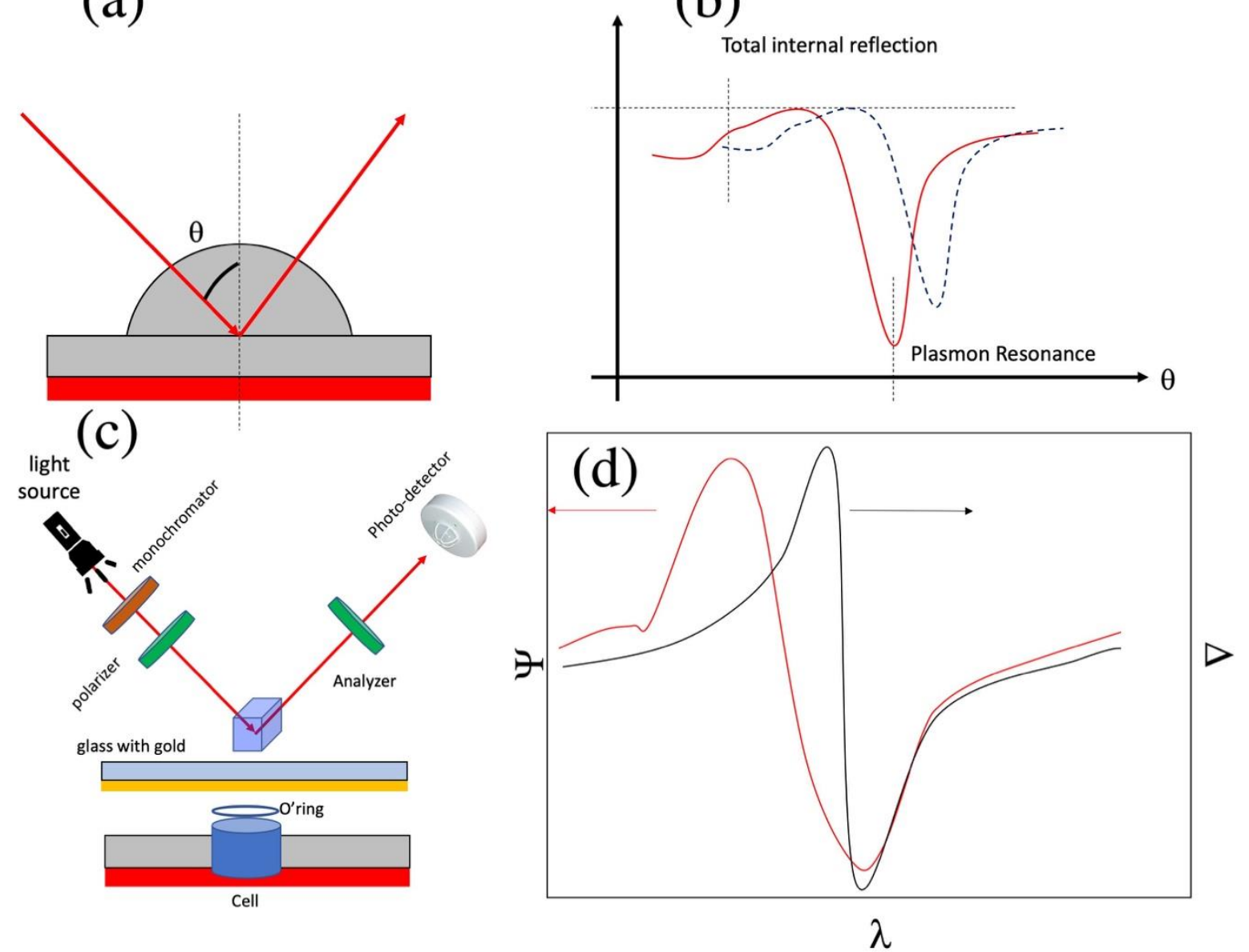

Figure 1. (a-b) An optical immunosensor is developed to determine aflatoxins in assay systems that generate specific absorbance when bound to antibodies immobilized in a sensitive optical component; (c-d) experimental apparatus, similar to an ellipsometer with the addition of a prism in optical contact with a gold-coated blade, and a typical spectrum of the TIRE method. 


\subsection{Biosensors based on the optical method.}

As previously described, mycotoxins are secondary metabolites produced by fungi [33]. Its universal character is due to the ease with which mycotoxin-producing fungi grow on a wide variety of substrates under different conditions, such as humidity, oxygenation, and temperature [34]. The main problem caused by mycotoxins is the degradation of agricultural products, which depends on environmental and storage conditions. It is estimated that mycotoxins contaminate around $25 \%$ of the world's grain production. Additionally, Eskola et al. presented a critical study of this data, as it has been widely cited since its publication in 1985 [35]. In their analysis, the authors concluded that the prevalence of mycotoxins strongly depends on the analytical methods used and that their incidence can reach $80 \%$ in some cases.

Mycotoxins have a wide variety of matrices with differences in their chemical structure, which leads us to a great challenge for their detection. Mycotoxins are often found as traces, so monitoring them requires instruments capable of detecting toxins at $\mathrm{ppb}$ and even ppt levels. Furthermore, it is possible to have the presence of more than one type of mycotoxin in the same sample. This requires a broad effort from the scientific community to develop and improve various analytical techniques for monitoring the different types of mycotoxins. Traditional methods, although efficient, are expensive and require laborious sample purification and protocols and trained personnel. Highly sensitive optical immunosensors are leading this development [36], as they are the most suitable for the detection of biotoxins, as they combine the high sensitivity of optical transducers with the high selectivity of immunological reactions.

The vast majority of optical detection methods are referenced to detect changes in the refractive index in the molecular layer, not to mention specific analytes. Instead of presenting the sensitivity, it is very common to be presented with the low detection limit (LDL) [37]. Another important parameter is related to the recovery levels and characteristics of the sensor times. These are related to response and recovery kinetics, depend on adsorption and desorption rates, and association and affinity constants. These parameters are important as they describe the specificity of the binding of the analyte molecule to the receptor.

The optical immunosensors are divided into two categories, luminescence sensors, where the sensing elements such as proteins, antibodies, enzymes, nanoparticles are combined with fluorescent markers. In this case, the answer can be easily visualized without the aid of robust equipment. An example of this method is the Enzyme-Linked Immunosorbent Assay (ELISA) [38]; this method has been established as a standard biosensing method in analytical laboratories and used as a comparative basis with other methods. The ELISA has a relatively high sensitivity, generally below $\mathrm{ng} / \mathrm{mL}$. However, the fact that it cannot be applied in the field and the use of expensive chemicals and high-test preparation time is its weak point.

The second category is based on label-free biosensors; most of these methods are based on the evanescent field phenomenon, which uses the change in the refractive index when an electromagnetic wave propagates between two different materials. The simplest form of this technique is simply a fiber optic tip coated with sensitive material; the intensity of the reflected light provides information about the interaction between the receptor and the analyte molecules. The method described above, although practical and easy to apply in the field, has limitations in terms of sensitivity, usually below ppm.

The most common optical biosensor is related to the surface plasmon resonance (SPR) phenomenon [39]. Figure 1(a-b) shows a classical Kretschmann SPR geometry. In this method, the evanescent wave propagates on the surface when the angle of incidence exceeds the critical 
angle of total internal reflection. However, if the wave vector $\mathrm{Kx}$ is equal to the vector Ks.p of the plasmons on the surface of thin metallic films, energy is transferred to the plasmons, and the reflection intensity drops. The relationship between the reflection intensity and the angle of incidence has an abrupt drop called surface plasma resonance (SRP), see Figure 1b. Since the evanescent wave penetrates beyond the metallic film, any molecular adsorption on the metallic surface will cause a shift in the SRP peak to high angles. These can be calibrated in terms of molecular concentrations. The designs for the SPR effect are the most varied; you can vary the angle of incidence, the wavelength of the incident light resulting in a spectroscopic SPR; portable SPR instruments have also been developed. The SPR detection has a detection sensitivity that can depend very much on the configuration used; the highest reported was 109 RIU achieved using special gold plates coated with a porous layer of $10 \mathrm{~nm}$ thicknesses of dextran, providing a wide range of detection surface. However, the most quoted values for SPR instruments are 3-4 orders of magnitude smaller.

Another widely used optical analytical method is ellipsometry, which is based on detecting changes in the polarization of light after its reflection from the surface to be studied [40]. Ellipsometry is a very sensitive analytical tool used to characterize various thin films and coatings with an accuracy of $0.01 \mathrm{~nm}$ for thickness and 10-5 for refractive index. In this method, the state of polarization of light is described by two ellipsometric parameters, $\Psi$ and $\Delta$ that represent the ratio of the amplitudes and the phase shift between the pes components of polarized light, $\Psi=\mathrm{Ap} /$ As and $\Delta=\varphi \mathrm{p}-\varphi \mathrm{s}$. These changes ( $\Psi$ and $\Delta$ ) are related to the optical parameters of the reflected surface through Fresnel equations. The presence of any thin film on the reflected surface, which may be a layer of adsorbed molecules, affects amplitude and phase values. Solving the Fresnel equations leads us to determine the adsorbed molecular layer's thickness and refractive index values from the measured values of $\Psi$ and $\Delta$. Thus, ellipsometry can be used to detect different chemical reactions on the surface. The main commercial ellipsometers, although they present high sensitivity, are difficult to apply as a biosensor. For its use, a cell has to be attached to the instrument allowing the insertion of different substances, both liquid and gaseous. Initially, they believed that the fact that light travels through the cell seemed to be the main obstacle to applying the sensor. Cells can be used relatively easily for gas detection but not for liquid detection. Performance is affected by different refractive index values in addition to the liquid medium absorbing or scattering light. Commercial cells are of high volume (tens of millimeters), which is not ideal for biodetection, normally using smaller volumes. This limitation was circumvented by combining the ellipsometry method with SPR [41], known as the total internal reflection (TIRE) ellipsometry method [42].

The TIRE method (Figure 1(c-d)) was developed by Professor A. Nabok's group and proved to be ideal for detecting low molecular weight analytes, such as mycotoxins[43]. The Figure below shows the experimental apparatus, similar to an ellipsometer with the addition of a prism with an angle of $68^{\circ}$ in optical contact with a gold-coated blade. A PTFE cell is sealed against the gold surface by means of a silicone O-ring; the cells have inlet and outlet tubes that allow the injection of different solutions. Figure 1(c-d) shows a typical spectrum of the TIRE method. The TIRE method is similar to traditional SPR with the difference that it uses a mixture of $\mathrm{p}$ and s polarized components instead of just $\mathrm{p}$ polarized light. The $\Delta$ spectrum represents a new amount of phase shift between the $\mathrm{p}$ and s components of polarized light, which does not exist in SPR. The method showed 10 times better sensitivity of the parameter $\Delta$ than of $\Psi$ to small changes in thickness and refractive index in the adsorbed molecular layer. Therefore, it is better to use spectra; the TIRE method can be termed as SPR phase. In SPR and ellipsometry, 
adjusting experimental data to Fresnels equations allows finding the optical parameters of thin molecular films, such as thickness (d) and complex refractive index $\mathrm{N}=\mathrm{n}-\mathrm{jk}$ (where $\mathrm{n}$ is the refractive index and $\mathrm{k}$ is the extinction coefficient ). However, the limitation of SPR and ellipsometry is that, for transparent dielectric films $(k=0, n>1)$ with thicknesses less than 10 $\mathrm{nm}$, it is impossible to find the values of $\mathrm{d}$ and $\mathrm{n}$ simultaneously.

A widespread method based on the evanescent field is optical spectroscopy in waveguide light mode (OWLS) [44]. It is similar to SPF with light coupled to a waveguide plate using a diffraction grating. The coupling angles depend on the type of light polarization used p-(TM) and s-(TE). These angles are recorded continuously by swinging the chip at a small angle $\pm 7 \mathrm{o}$, and the two peaks of coupling intensity are recorded at angles $\alpha$ s and $\alpha \mathrm{p}$. If the adsorption of molecules takes place at the top of the waveguide, the positions of both the $\alpha$ s and $\alpha$ p peaks shift to higher angles. As s-polarized light is less affected than p-polarization, changes in the adsorbed molecular layer make the difference between the coupling angles $\Delta=$ $\alpha$ s - $\alpha p$ can be used as a sensor response calibrated in concentrations of adsorbed molecules. The OWLS method has a similar sensitivity to the SPR method, in the range of 10-6 RIU. The method in question has an additional advantage; the exact solution of the model equations allows the simultaneous determination of $\mathrm{n}$ and $\mathrm{d}$ of the adsorbed molecular layer. However, it has a disadvantage because the experimental apparatus has a large volume, is relatively expensive, and cannot be applied in loco.

Even as the interferometric methods are widely used as very sensitive analytical tools in optics, we have a dual-polarization interferometer (DPI) [45]. The DPI works based on the interference of two waves that propagate in adjacent layers and on the formation of the interference pattern. Once the upper waveguide plate is exposed to the environment, the molecular adsorption that affects the propagation wave causes the interference pattern to shift, which can be quantified in the concentrations of adsorbed molecules. This method has a sensitivity of $10^{-7}$ RIU, comparable to that presented by the SPR method. This method presents a direct analysis of the results, easy to interpret, depending only on the change of parameters such as refractive index or thickness or optical density of the molecular layer. One downside is its high value, and it is not possible to analyze it in the field.

One of the most promising instruments for biosensing application is the Mach Zehnder interferometer [46]. This technique uses an optoelectronic device to mix and modulate optical signals. An opening in the case of one of the arms acts as a sensing element, while the second arm serves as a reference. The interferometer presents a multiperiod signal proportional to the phase shift between the two arms: R sins-r, where the indices s and $\mathrm{r}$ represent the detection and reference arms. The MZ interferometer has a sensitivity in the range of $10^{-7}-10^{-9}$ RIU.

In 1908, Mie was the first to observe the phenomenon of localized surface plasmon resonance (LSPR), a promising technique for application as a biosensor [47]. The LSPR phenomenon is caused by oscillations of electron surfaces normally in metallic nanostructures, such as nanoparticles with dimensions in the order of the wavelength of light. The LSPR effect is easily detectable using a standard spectrophotometer. The position and shape of the peak strongly depend on the refractive index of the surrounding medium. Fulano et al. showed that the refractive index $\left(n_{m}\right)$ causes a shift in peak $\left(\lambda_{L S P R}\right)$ for longer wavelengths in relation to the plasmon peak in the bulk material $\left(\lambda_{0}\right): \lambda_{L S P R}=\lambda_{0} \sqrt{2 n a_{m}^{2}+1}$. The previous paragraph describes the principle of sensors based on the LSPR phenomenon. The molecules adsorbed on the surface of the metallic nanostructures change the local refractive index in the vicinity of the 
metallic nanoparticles, changing the adsorption band that can then be quantified and calibrated against the concentration of the adsorbed molecules.

Another method used for biodetection is surface-enhanced Raman scattering (SERS), long known as the method of substantially increasing the sensitivity of Raman spectroscopy using rough surfaces due to the scattering of electromagnetic waves and the associated local increase in the electric field [48]. The SERS effect can be particularly useful for chemical detection. Biotoxins of different types can be identified by their characteristic vibration spectrum signatures; in this case, the detection sensitivity could actually go down to the level of a single molecule. However, SERS acts in a very narrow spectrum band and can only increase specific vibration bands. Recently, the SERS of "band-gap" was observed, which provides great resolution with accent $10^{5}-10^{6}$ in a wide spectrum range, covering virtually the entire infrared spectrum.

\subsection{Electrochemical biosensor.}

Amperometric immunosensors have become a widely used technique for detecting contaminants in grain, mainly based on the ELISA technique [48]. With the advantages of fast, sensitive, and selective quantification - when functionalized, this method has been employed to determine mycotoxins without the requirement of complex sample preparation as optical methods. These sensors are gauged based on chronoamperometry techniques; a constant electrical potential is applied, and the electrical current is analyzed. Arevalo et al. constructed an electrochemical microfluidic immunosensor to detect citrinin in rice samples [49]. The advantage of this type of biosensors is that they can be produced on demand and applied in loco.

Another class of electrochemical sensors is potentiometric. Both differential pulse voltammetry (DPV) and cyclic voltammetry (CV) are employed to detect mycotoxins. Differential pulse voltammetry (DPV) reduces the effect of load current on sample measurement by sensing the current before the potential change. DPV can serve to detect single mycotoxins and multiple mycotoxins [50]. An application of DPV biosensors used an electrochemical aptamer sensor to detect ochratoxin in a wheat starch sample, reaching a detection limit of $1.0 \mathrm{pg} / \mathrm{mL}$. DPV was obtained with signal amplification, which was achieved by the simultaneous formation and release of the aptamer-ochratoxin cluster with exonuclease consumption. The variation in the signal before and after the addition of ochratoxin was detected to quantify this analyte. The cyclic voltammetry method has also been used to detect mycotoxins. Technique in which a potential is applied to the working electrode and the originating electrical current is measured. Hervas et al. presented a study presenting a dual T microchip for monitoring zearalenone in infant foods [51]. In the experiment, the authors showed a negative correlation between the signal and concentration of mycotoxin. Based on competitive binding between zearalenone and enzyme-labeled derivatives to a specific antibody, quantitative detection was achieved by adding electrochemical mediators and enzyme substrates. By using both channels as immune and enzymatic reaction sites, nonspecific adsorption can be avoided.

\subsection{Nanomaterial-based sensors.}

With the advent and development of certain researches towards miniaturization - as in the case of microelectronics - a variety of materials reduced to a nanometer level (0.1-100 
nanometer) have been designed and prepared, such as carbon nanomaterials (carbon nanotubes and graphene), metal nanoparticles, nanowires, nanocomposites, and nanostructured metal oxide nanoparticles. These nanoparticles or nanomaterials - when associated with biosensors show potential and properties in integrated devices, such as increased sensitivity and accuracy in relation to measured data, amplification of the transmitted signal, reduction of sample preparation, in addition, of course, to the miniaturization of devices. In this context, nanobiosensors are classified according to the type of nanoparticle that constitutes it, in addition to its advantages and limitations conditioned to the inherent properties of the material used.

The biosensors mentioned above are used in the detection of aflatoxin B1. Although immunosensors and aptamer integrated electrochemical are potentially more sensitive and consistent in detecting B1, they are cumbersome and non-specific due to the binding of nontarget molecules with immobilized antibodies and require qualified personnel and device for their operation, respectively. Impedimetric biosensors are fast methods, but their detection processes are expensive and unreliable due to the presence of electrolytes in the solution. Potentiometric biosensors have a simple and very sensitive detection but depend on other parameters such as $\mathrm{pH}$ and temperature. Colorimetric biosensors are fast, sensitive, and simple but are less reproducible and sometimes give false-positive results. Conductometric biosensors are economical but exhibit limited sensitivity and accuracy and can measure pseudo results.

\section{Fungal Toxins in Stored Agricultural Products}

\subsection{Regulations.}

The concentration of mycotoxins found in products of agricultural origin does not produce acute toxic effects in consumers or farm animals [52,53]. Differently, chronic exposure or consumption of adults according to substances can lead to health risks due to the frequent consumption of material contaminated with casualties, leading to the appearance of health problems[54,55].

Socio-economic factors such as the level of economic development and food security of a country or region are important components of regulations that protect consumers against foods contaminated by fungal toxins. Assessments are built based on available information about their toxicity and transport potential by animals that ingest these molecules.

In products of plant origin, to establish tolerable levels, limits of mycotoxins in food and feed are defined by different sanitary and regulatory authorities of agricultural production, such as the Food and Drug Administration (FDA) of the United States, the World Health Organization (WHO), a Food Agriculture Organization (FAO) and the European Food Safety Authority (EFSA) [54,55]. Compliance with acceptable limits for mycotoxins is the responsibility of the Codex Alimentarius Commissions (CAC). The objective of harmonizing acceptable mycotoxin limits is to facilitate world trade in agricultural commodities and prevent the exposure of humans and animals to unacceptable levels of mycotoxins[56]. More than 168 countries are currently members of Codex Alimentarius and follow methods developed in the Codex Manual of Procedures for Mycotoxin Regulations and their Acceptable Limits in Grains, Food, and Animal Feed[57].

The International Agency for Research on Cancer (IARC) categorizes some mycotoxins by evidence of carcinogenicity through toxicological studies in humans. Rapid Alert System for Food and Feed (RASFF) monitors the contamination of food and feed by 
mycotoxins in Europe [58]. Through RASFF, all EU Member States can be informed through an information exchange system and measures to ensure food and feed safety [59]. In 2019 RASFF reported 534 notifications of mycotoxins in food, most related to the presence of aflatoxins in products such as nuts and grains, cereals, fruits, and vegetables, or herbs and seasonings [58]. Developed countries with more elaborate scientific and technical knowledge tend to adopt stricter regulatory limits than those created by food safety regulatory bodies [52].

With the assurance of consumer protection, many locations set maximum limits for aflatoxins and other mycotoxins in food and feed. In the United States, the FDA elevated a maximum concentration of $10-15 \mathrm{ppb}$ of total aflatoxins (in products such as hazelnuts, almonds, pistachios, and Brazil nuts) and $0.5 \mathrm{ppb}$ of AFM1 in whole milk and dairy products [60]. In the European Union, the standard levels range from 0.1 to $12 \mathrm{ppb}$ for AFB1; 4-15 ppb for total aflatoxins, and 0.025-0.05 ppb for AFM1. FDA allows a maximum of $300 \mathrm{ppb}$ of total aflatoxins in cottonseed meal, corn, and peanut products when these raw materials are used in finishing beef cattle, swine, and poultry: $100 \mathrm{ppb}$ for reproduction and $20 \mathrm{ppb}$ for other animals (https://www.fda.gov/media/121202/download). In the European Union, the limits for AFB1 vary between 5 and $20 \mathrm{ppb}$ for the materials used in animal feed.

In Latin America, several countries, including non-Mercosur group members, have enacted regulations to prevent aflatoxins in food and feed [61]. Almost all states in Asia have written regulations for aflatoxin, mainly for cereals, nuts, and their products [62]. Gulf Cooperation Council members (United Arab Emirates, Saudi Arabia, Qatar, Oman, Kuwait, Bahrain) have also jointly adopted regulations related to aflatoxin [63]. Although the high occurrence of aflatoxins is frequently reported in Africa, only a few countries have adopted regulations for these substances in food. Among them are Nigeria, Côte d'Ivoire, Senegal, Egypt, Morocco, Tunisia, South Africa, Kenya, and Zimbabwe [64].

\section{Conclusions}

To explore the chemical and structural properties of agricultural products and foods contaminated by aflatoxins, adaptations in the means to detect and quantify these mycotoxins have been adapted. It is still necessary to increase the effectiveness of aflatoxin measurements in these products at the production and storage site and to specify this, and it is necessary to improve the current knowledge of aspects of specific products in order to detect aflatoxins in a more precise and simple way. So far, it has been highlighted that the main methods used to determine significant levels of aflatoxins are chromatographic, immunochemical, and spectroscopic. However, new methods are needed to increase the specificity of detection in agricultural products and foods with rapid analysis techniques that would lead to safe food consumption.

\section{Funding}

This research received no external funding.

\section{Acknowledgments}

Cleber A. Amorim would like to thank the scholarship granted by FAPESP 2019/18963-6 in this research topic. 


\section{Conflicts of Interest}

The authors declare no conflict of interest.

\section{References}

1. Negash, D. A review of aflatoxin: occurrence, prevention, and gaps in both food and feed safety. J. Nutr. Heal. Food Eng. 2018, https://doi.org/10.15406/jnhfe.2018.08.00268.

2. Bennett, J.W. Mycotoxins, mycotoxicoses, mycotoxicology and Mycopathologia. Mycopathologia 1987.

3. Taniwaki, M.H.; Hocking, A.D.; Pitt, J.I.; Fleet, G.H. Growth and mycotoxin production by fungi in atmospheres containing $80 \%$ carbon dioxide and $20 \%$ oxygen. Int. J. Food Microbiol. 2010, https://doi.org/10.1016/j.ijfoodmicro.2010.08.030.

4. Paterson, R.R.M.; Lima, N. How will climate change affect mycotoxins in food? Food Res. Int. 2010, https://doi.org/10.1016/j.foodres.2009.07.010.

5. Silva, J.V.B. Da; Oliveira, C.A.F. De; Ramalho, L.N.Z. An overview of mycotoxins, their pathogenic effects, foods where they are found and their diagnostic biomarkers. Food Sci. Technol. 2021, https://doi.org/10.1590/fst.48520.

6. Logrieco, A.; Bottalico, A.; Mulé, G.; Moretti, A.; Perrone, G. Epidemiology of toxigenic fungi and their associated mycotoxins for some Mediterranean crops. Eur. J. Plant Pathol. 2003.

7. Singh, V.K.; Meena, M.; Zehra, A.; Tiwari, A.; Dubey, M.K.; Upadhyay, R.S. Fungal toxins and their impact on living systems. In Microbial Diversity and Biotechnology in Food Security 2014.

8. Santin, E.; Maiorka, A.; Zanella, I.; Magon, L. Micotoxinas do fusarium spp na avicultura comercial. Ciência Rural 2001, https://doi.org/10.1590/s0103-84782001000100030.

9. Diggins, F.W.E. The true history of the discovery of penicillin, with refutation of the misinformation in the literature. Br. J. Biomed. Sci. 1999.

10. Oancea, S.; Stoia, M. Mycotoxins: a review of toxicology, analytical methods and health risks - review - . Acta Univ. Cibiniensis Ser. E FOOD Technol. 2008.

11. PITT, J.I. Corrections to Species Names in Physiological Studies on Aspergillus flavus and Aspergillus parasiticus. J. Food Prot. 1993, https://doi.org/10.4315/0362-028x-56.3.265.

12. LACEY, J. Water availability and the occurrence of toxigenic fungi and mycotoxins in stored products. Mycotoxins 1988, https://doi.org/10.2520/myco1975.1988.1supplement_186.

13. Diener, U.L.; Davis, N.D. Limiting temperature and relative humidity for growth and production of aflatoxin and free fatty acids by Aspergillus flavus in sterile peanuts. J. Am. Oil Chem. Soc. 1967, https://doi.org/10.1007/BF02639271.

14. Dickens, F.; Jones, H.E. The carcinogenic action of aflatoxin after its subcutaneous injection in the rat. $B r . J$. Cancer 1963, https://doi.org/10.1038/bjc.1963.88.

15. Castegnaro, M.; McGregor, D. Carcinogenic risk assessment of mycotoxins. Rev. Med. Vet. (Toulouse). 1998.

16. Svoboda, D.; Grady, H.J.; Higginson, J. Aflatoxin B1 injury in rat and monkey liver. Am. J. Pathol. 1966.

17. Shank, R.C.; Johnsen, D.O.; Tanticharoenyos, P.; Wooding, W.L.; Bourgeois, C.H. Acute toxicity of aflatoxin B1 in the macaque monkey. Toxicol. Appl. Pharmacol. 1971, https://doi.org/10.1016/0041$008 X(71) 90048-2$.

18. Souza, M.F.; Tomé, A.R.; Rao, V.S.N. Inhibition by the Bioflavonoid Ternatin of Aflatoxin B1-induced Lipid Peroxidation in Rat Liver. J. Pharm. Pharmacol. 2010, https://doi.org/10.1211/0022357991772222.

19. Bailey, E.A.; Iyer, R.S.; Stone, M.P.; Harris, T.M.; Essigmann, J.M. Mutational properties of the primary aflatoxin B1-DNA adduct. Proc. Natl. Acad. Sci. U. S. A. 1996, https://doi.org/10.1073/pnas.93.4.1535.

20. Schrenk, D.; Bignami, M.; Bodin, L.; Chipman, J.K.; del Mazo, J.; Grasl-Kraupp, B.; Hogstrand, C.; Hoogenboom, L.; Leblanc, J.C.; Nebbia, C.S.; et al. Risk assessment of aflatoxins in food. EFSA J. 2020, https://doi.org/10.2903/j.efsa.2020.6040.

21. Kimanya, M.E.; Routledge, M.N.; Mpolya, E.; Ezekiel, C.N.; Shirima, C.P.; Gong, Y.Y. Estimating the risk of aflatoxin-induced liver cancer in Tanzania based on biomarker data. PLoS One 2021, https://doi.org/10.1371/journal.pone.0247281.

22. Yu, J.; Hennessy, D.A.; Wu, F. The Impact of Bt Corn on Aflatoxin-Related Insurance Claims in the United States. Sci. Rep. 2020, https://doi.org/10.1038/s41598-020-66955-1.

23. A Ogungbemile, O.; M Etaware, P.; C Odebode, A. Aflatoxin Detection and Quantification in Stored Cowpea Seeds in Ibadan, Nigeria. J. Biotechnol. Biomed. 2020, https://doi.org/10.26502/jbb.2642-91280022.

24. Ahmed Abdullah Murshed, S.; Bacha, N.; Alharazi, T. Detection of Total Aflatoxins in Groundnut and Soybean Samples in Yemen Using Enzyme-Linked Immunosorbent Assay. J. Food Qual. 2019, https://doi.org/10.1155/2019/1614502.

25. Tarannum, N.; Nipa, M.N.; Das, S.; Parveen, S. Aflatoxin M1 detection by ELISA in raw and processed milk in Bangladesh. Toxicol. Reports 2020, https://doi.org/10.1016/j.toxrep.2020.09.012.

26. Algammal, A.M.; Elsayed, M.E.; Hashem, H.R.; Ramadan, H.; Sheraba, N.S.; El-Diasty, E.M.; Abbas, S.M.; Hetta, H.F. Molecular and HPLC-based approaches for detection of aflatoxin B1 and ochratoxin A released from toxigenic Aspergillus species in processed meat. BMC Microbiol. 2021, https://doi.org/10.1186/s12866- 
021-02144-y.

27. Zhao, F.; Tian, Y.; Shen, Q.; Liu, R.; Shi, R.; Wang, H.; Yang, Z. A novel nanobody and mimotope based immunoassay for rapid analysis of aflatoxin B1. Talanta 2019, https://doi.org/10.1016/j.talanta.2018.11.013.

28. Gichohi-Wainaina, W.N.; Kumwenda, N.; Zulu, R.; Munthali, J.; Okori, P. Aflatoxin contamination: Knowledge disparities among agriculture extension officers, frontline health workers and small holder farming households in Malawi. Food Control 2021, https://doi.org/10.1016/j.foodcont.2020.107672.

29. Pickova, D.; Ostry, V.; Toman, J.; Malir, F. Aflatoxins: History, significant milestones, recent data on their toxicity and ways to mitigation. Toxins (Basel) 2021.

30. Bertani, F.R.; Businaro, L.; Gambacorta, L.; Mencattini, A.; Brenda, D.; Di Giuseppe, D.; De Ninno, A.; Solfrizzo, M.; Martinelli, E.; Gerardino, A. Optical detection of aflatoxins B in grained almonds using fluorescence spectroscopy and machine learning algorithms. Food Control 2020, https://doi.org/10.1016/j.foodcont.2019.107073.

31. Righetti, L.; Bhandari, D.R.; Rolli, E.; Tortorella, S.; Bruni, R.; Dall'Asta, C.; Spengler, B. Unveiling the spatial distribution of aflatoxin B1 and plant defense metabolites in maize using AP-SMALDI mass spectrometry imaging. Plant J. 2021, https://doi.org/10.1111/tpj.15158.

32. Singh, A.K.; Dhiman, T.K.; Lakshmi, V.S.G.B.; Solanki, P.R. Dimanganese trioxide (Mn2O3) based labelfree electrochemical biosensor for detection of Aflatoxin-B1. Bioelectrochemistry 2021, https://doi.org/10.1016/j.bioelechem.2020.107684.

33. Vanhoutte, I.; Audenaert, K.; De Gelder, L. Biodegradation of mycotoxins: Tales from known and unexplored worlds. Front. Microbiol. 2016, 7.

34. Medeiros, F.H.V.; Martins, S.J.; Zucchi, T.D.; Melo, I.S.; Batista, L.R.; Machado, J. da C. Biological control of mycotoxin-producing molds. Ciência e Agrotecnologia 2012, 36, https://doi.org/10.1590/s141370542012000500001 .

35. Eskola, M.; Kos, G.; Elliott, C.T.; Hajšlová, J.; Mayar, S.; Krska, R. Worldwide contamination of food-crops with mycotoxins: Validity of the widely cited 'FAO estimate' of 25\%. Crit. Rev. Food Sci. Nutr. 2020, 60.

36. Ramírez, N.B.; Salgado, A.M.; Valdman, B. The evolution and developments of immunosensors for health and environmental monitoring: Problems and perspectives. Brazilian J. Chem. Eng. 2009, 26, https://doi.org/10.1590/s0104-66322009000200001.

37. Andrade, C.; Danielly, M.; Faulin, T.; Hering, V.; Parra Abdall, D.S. Biosensors for detection of Low-Density Lipoprotein and its modified forms. In Biosensors for Health, Environment and Biosecurity; 2011.

38. Engvall, E.; Perlmann, P. Enzyme-linked immunosorbent assay (ELISA) quantitative assay of immunoglobulin G. Immunochemistry 1971, 8, https://doi.org/10.1016/0019-2791(71)90454-X.

39. Souto, D.E.P.; Volpe, J.; Gonçalves, C. de C.; Ramos, C.H.I.; Kubota, L.T. A brief review on the strategy of developing SPR-based biosensors for application to the diagnosis of neglected tropical diseases. Talanta 2019, 205, https://doi.org/10.1016/j.talanta.2019.120122.

40. Grieshaber, D.; MacKenzie, R.; Vörös, J.; Reimhult, E. Electrochemical biosensors - Sensor principles and architectures. Sensors 2008, 8.

41. Xiao, C. SPR ellipsometry biosensors. In Super Optical Biosensors 2019.

42. Balevicius, Z.; Baskys, A. Optical dispersions of bloch surface waves and surface plasmon polaritons: Towards advanced biosensors. Materials (Basel). 2019, 12, https://doi.org/10.3390/ma12193147.

43. Nabok, A.; Tsargorodskaya, A.; Mustafa, M.K.; Székács, I.; Starodub, N.F.; Székács, A. Detection of low molecular weight toxins using an optical phase method of ellipsometry. In Proceedings of the Sensors and Actuators, B: Chemical 2011, 154.

44. Kłos-Witkowska, A. The phenomenon of fluorescence in immunosensors. Acta Biochim. Pol. $2016,63$.

45. Peltomaa, R.; Glahn-Martínez, B.; Benito-Peña, E.; Moreno-Bondi, M.C. Optical Biosensors for Label-Free Detection of Small Molecules. Sensors (Basel) 2018, 18.

46. Janik, M.; Koba, M.; Celebańska, A.; Bock, W.J.; Śmietana, M. Live E. coli bacteria label-free sensing using a microcavity in-line Mach-Zehnder interferometer. Sci. Rep. 2018, 8, https://doi.org/10.1038/s41598-01835647-2.

47. Wei, T.; Ren, P.; Huang, L.; Ouyang, Z.; Wang, Z.; Kong, X.; Li, T.; Yin, Y.; Wu, Y.; He, Q. Simultaneous detection of aflatoxin B1, ochratoxin A, zearalenone and deoxynivalenol in corn and wheat using surface plasmon resonance. Food Chem. 2019, 300, https://doi.org/10.1016/j.foodchem.2019.125176.

48. Liu, D.; Li, W.; Zhu, C.; Li, Y.; Shen, X.; Li, L.; Yan, X.; You, T. Recent progress on electrochemical biosensing of aflatoxins: A review. TrAC - Trends Anal. Chem. 2020, 133.

49. Fernández, H.; Arévalo, F.J.; Granero, A.M.; Robledo, S.N.; Nieto, C.H.D.; Riberi, W.I.; Zon, M.A. Electrochemical biosensors for the determination of toxic substances related to food safety developed in south America: Mycotoxins and herbicides. Chemosensors 2017, 5.

50. Wang, C.; Zhang, H.; Jiang, X.; Zhou, B. Electrochemical Determination of Aflatoxin B1 (AFB1) Using a Copper-Based Metal-Organic Framework (Cu-MOF) and Gold Nanoparticles (AuNPs) with Exonuclease III (Exo III) Assisted Recycling by Differential Pulse Voltammetry (DPV). Anal. Lett. 2019, 52, https://doi.org/10.1080/00032719.2019.1610418.

51. Hervás, M.; López, M.A.; Escarpa, A. Integrated electrokinetic magnetic bead-based electrochemical immunoassay on microfluidic chips for reliable control of permitted levels of zearalenone in infant foods. 
Analyst 2011, 136, https://doi.org/10.1039/c1an15081b.

52. Ibrahim, O.O.; Menkovska, M. The Nature, Sources, Detections and Regulations of Mycotoxins That Contaminate Foods and Feeds Causing Health Hazards for Both Human and Animals. J. Agric. Chem. Environ. 2019, https://doi.org/10.4236/jacen.2019.81004.

53. Buszewska-Forajta, M. Mycotoxins, invisible danger of feedstuff with toxic effect on animals. Toxicon 2020.

54. Schaarschmidt, S.; Fauhl-Hassek, C. The fate of mycotoxins during the primary food processing of maize. Food Control 2021.

55. Azam, M.S.; Ahmed, S.; Islam, M.N.; Maitra, P.; Islam, M.M.; Yu, D. Critical assessment of mycotoxins in beverages and their control measures. Toxins (Basel). 2021.

56. Atanda S. A Fungi and mycotoxins in stored foods. African J. Microbiol. Res. 2011, https://doi.org/10.5897/ajmr11.487.

57. Suman, M. Last decade studies on mycotoxins' fate during food processing: an overview. Curr. Opin. Food Sci. 2021.

58. Fuentes, V.R. The rapid alert system for food and feed - A critical approach. Eur. Food Feed Law Rev. 2017.

59. Kowalska, A.; Manning, L. Using the rapid alert system for food and feed: potential benefits and problems on data interpretation. Crit. Rev. Food Sci. Nutr. 2021.

60. Cheng, C. Codex alimentarius commission. In Encyclopedia of Food Security and Sustainability 2018.

61. Martinez, M.; Vargas, L.; Gomez, V. Aflatoxinas: incidencia, impactos en la salud, control y prevención. Biosalud 2013.

62. Anukul, N.; Vangnai, K.; Mahakarnchandkul, W. Significance of regulation limits in mycotoxin contamination in Asia and risk management programs at the national level. J. Food Drug Anal. 2013.

63. Al-Jaal, B.; Salama, S.; Al-Qasmi, N.; Jaganjac, M. Mycotoxin contamination of food and feed in the Gulf Cooperation Council countries and its detection. Toxicon 2019.

64. Chauhan, N.M. Aflatoxin: A Risky Menace for African's Food Commodities. In Aflatoxin-Control, Analysis, Detection and Health Risks 2017. 